\title{
REMARKS ON A PAPER BY ZEEV NEHARI
}

\section{EINAR HILLE}

In the preceding paper Zeev Nehari has proved some interesting inequalities for the Schwarzian derivative of a univalent (=schlicht) function. Thus if $f(z)$ is univalent in the unit circle, then

$$
|\{f(z), z\}| \leqq 6\left[1-|z|^{2}\right]^{-2}
$$

while if

$$
|\{f(z), z\}| \leqq 2\left[1-|z|^{2}\right]^{-2},
$$

then $f(z)$ is univalent for $|z|<1$. The object of the present note is to show that 2 is the best possible constant in (2) in the following sense:

For every $C>2$ there exists a function $f(z)$ such that for $|z|<1$ we have (i) $f(z)$ is holomorphic, (ii) $f(z)$ takes on the value one infinitely often, and (iii) $|\{f(z), z\}| \leqq C\left[1-|z|^{2}\right]^{-2}$ with equality for real values of $z$.

An explicit example of such a function is given by

$$
f(z)=\left(\frac{1-z}{1+z}\right)^{\gamma i}
$$

where $\gamma$ is a real constant, $f(0)=1$, and $C=2\left(1+\gamma^{2}\right)$.

In view of the background of the problem, the following approach is natural. Let $F_{a}$ denote the family of fractional linear transforms with constant coefficients of the quotient of two linearly independent solutions of the differential equation,

$$
\frac{d^{2} y}{d z^{2}}+a\left(1-z^{2}\right)^{-2} y=0,
$$

where $a$ is an arbitrary parameter. If $f(z) \in F_{a}$, then

$$
\{f(z), z\}=2 a\left(1-z^{2}\right)^{-2} .
$$

If one function $f(z)$ of $F_{a}$ is univalent in the unit circle, then they all are. Let us determine the region $U$ of the $a$-plane such that if $a \in U$, then the functions of $F_{a}$ are univalent for $|z|<1$. By Nehari's Theorem I the region $|a| \leqq 1$ belongs to $U$ and (1) makes it plausible that $U$ is contained in $|a| \leqq 3$.

Equation (4) has elementary solutions. The indicial equations at 1948.

Presented to the Society, September 10, 1948; received by the editors July 16, 
the singular points $z= \pm 1$ are identical,

$$
\rho^{2}-\rho+\frac{1}{4} a=0,
$$

and if its two roots are $\alpha$ and $\beta$, a simple calculation shows that

$$
y(z)=(1-z)^{\alpha}(1+z)^{\beta}
$$

is a solution. If $a \neq 1, \alpha \neq \beta$ and $y(-z)$ is a second linearly independently solution. The family $F_{a}$ is generated by their quotient

$$
f(z)=\left(\frac{1-z}{1+z}\right)^{\delta}, \quad \delta=\beta-\alpha=(1-a)^{1 / 2}=\mu+i \nu
$$

where $f(0)=1$ and the square root is +1 for $a=0$.

It remains to determine when $f(z)$ is univalent in $|z|<1$ or, what is equivalent, when $t^{\delta}$ is univalent in the right half-plane. A necessary and sufficient condition that for every choice of $v$ the equation $t^{\delta}=v$ shall have at most one solution with $|\arg t|<\frac{1}{2} \pi$ is given by $2|\mu|$ $\geqq \mu^{2}+\nu^{2}$. This implies that either $|\delta-1| \leqq 1$ or $|\delta+1| \leqq 1$. Since $a=1-\delta^{2}$, we see that the boundary of $U$ is the cardioid

$$
a=-2 e^{i \phi}-e^{2 i \phi}, \quad-\pi<\phi \leqq \pi,
$$

and $U$ consists of the interior and boundary of the cardioid. We note that the largest circle with center at the origin contained in $U$ is $|a|=1$ and that $|a| \leqq 3$ in $U$ with equality only for $a=-3$.

Every point $a$ outside of $U$ leads to a family of functions which are not univalent in the unit circle. In particular, for every real $\gamma, \gamma \neq 0$, the point $a=1+\gamma^{2}$ is outside of $U$. Thus the function (3) is not univalent for $|z|<1$ and a simple calculation shows that it takes on the value one infinitely often in the unit circle.

In conclusion, we observe that the boundary point $a=-3$ of $U$ is of considerable interest. One of the functions of $F_{-3}$ is $4 z(1+z)^{-2}$ which is the extremal function of Koebe's mapping problem.

YALE UNIVERSity 